$\begin{array}{lllll}\text { C1 } & 0.0953(2) & 0.4610(2) & 0.2955(4) & 0.0493(6) \\ \text { C2 } & 0.2009(2) & 0.4990(2) & 0.2218(4) & 0.0531(6) \\ \text { C3 } & 0.2141(2) & 0.6101(2) & 0.1942(4) & 0.0548(7) \\ \text { C4 } & 0.1227(2) & 0.6799(2) & 0.2399(4) & 0.0565(7) \\ \text { C5 } & 0.0183(2) & 0.6428(2) & 0.3166(5) & 0.0622(8) \\ \text { C6 } & 0.0054(2) & 0.5316(2) & 0.3461(4) & 0.0565(7) \\ \text { C7 } & 0.1531(2) & 0.2788(2) & 0.3783(4) & 0.0555(7) \\ \text { C8 } & 0.0117(3) & 0.1424(3) & 0.2588(7) & 0.0814(11) \\ \text { C9 } & 0.1906(4) & 0.0890(3) & 0.4371(7) & 0.0845(11)\end{array}$

Sheldrick, G. M. (1993). SHELXL93. Program for Crystal Structure Refinement. University of Göttingen, Germany.

Zsolnai, L. (1994). ZORTEP. Interactive Graphics Program. University of Heidelberg, Germany.

Acta Cryst. (1996). C52, 104-107

\section{A Pinacol Precursor to a Chiral Spiro Compound}

$1.374(4)$

$1.375(4)$

$1.381(4)$

$\mathrm{Nl}-\mathrm{C} 9 \mathrm{O} \quad 1.454(5)$

$\mathrm{Ni}-\mathrm{C} 8$

$\mathrm{C} 7-\mathrm{O} 1-\mathrm{Cl}$

$1.457(4)$

$\mathrm{C} 7-\mathrm{Nl}-\mathrm{C} 9$

$\mathrm{C} 7-\mathrm{N} 1-\mathrm{C} 8 \quad 124.2(3)$

$\mathrm{C} 9-\mathrm{N} 1-\mathrm{C} 8 \quad 117.2(3)$

$\mathrm{O} 3-\mathrm{N} 2-\mathrm{O} 4 \quad 123.7(3)$

$\mathrm{O} 3-\mathrm{N} 2-\mathrm{C} 4 \quad 118.4(3)$

$\mathrm{O} 4-\mathrm{N} 2-\mathrm{C} 4 \quad 118.0(3)$

$\mathrm{C} 6-\mathrm{Cl}-\mathrm{C} 2$

$\mathrm{C} 6-\mathrm{Cl}-\mathrm{Ol} \quad 115.9(2)$

$\mathrm{C} 2-\mathrm{Cl}-\mathrm{O} 1$

$122.4(2)$

$\begin{array}{ll}\mathrm{C} 3-\mathrm{C} 2-\mathrm{Cl} & 118.6(2) \\ \mathrm{C} 4-\mathrm{C} 3-\mathrm{C} 2 & 119.5(2) \\ \mathrm{C} 3-\mathrm{C} 4-\mathrm{C} 5 & 122.1(2) \\ \mathrm{C} 3-\mathrm{C} 4-\mathrm{N} 2 & 119.0(3) \\ \mathrm{C} 5-\mathrm{C} 4-\mathrm{N} 2 & 118.9(3) \\ \mathrm{C} 4-\mathrm{C} 5-\mathrm{C} 6 & 118.4(3) \\ \mathrm{C} 1-\mathrm{C} 6-\mathrm{C} 5 & 119.7(3) \\ \mathrm{O} 2-\mathrm{C} 7-\mathrm{N} 1 & 126.3(3) \\ \mathrm{O} 2-\mathrm{C} 7-\mathrm{O} 1 & 122.8(2) \\ \mathrm{N} 1-\mathrm{C} 7-\mathrm{O} 1 & 110.8(2)\end{array}$

For both (1) and (2), $\mathrm{H}$ atoms were placed in calculated sites riding on the $\mathrm{C}$ atoms to which they were attached. Isotropic displacement parameters were fixed at $0.08 \AA^{2}$.

For both compounds, data collection: $C A D-4$ Software (Enraf-Nonius, 1989); cell refinement: CAD-4 Software; data reduction: MolEN (Fair, 1990); program(s) used to solve structures: SHELXS86 (Sheldrick, 1985); program(s) used to refine structures: SHELXL93 (Sheldrick, 1993); molecular graphics: ZORTEP (Zsolnai, 1994); software used to prepare material for publication: SHELXL93.

The financial assistance of Massey University and New Zealand Lottery Science is gratefully acknowledged.

Lists of structure factors, anisotropic displacement parameters, $\mathrm{H}$ atom coordinates and complete geometry have been deposited with the IUCr (Reference: TA1035). Copies may be obtained through The Managing Editor, International Union of Crystallography, 5 Abbey Square, Chester $\mathrm{CH} 12 \mathrm{HU}$, England.

\section{References}

Baker, H. M., Blackwell, L. F., Brown, R. L., Buckley, P. D., Dobbs, A. J., Hardman, M. J., Hill, J. P., Kitson, K. E., Kitson, T. M. \& Baker, E. N. (1994). J. Mol. Biol. 241, 263-264.

Burr, M. \& Koshland, D. E. (1964). Proc. Natl Acad. Sci, USA, 52, 1017-1024.

Enraf-Nonius (1989). CAD-4 Software. Version 5.0. Enraf-Nonius, Delft, The Netherlands.

Fair, C. K. (1990). MolEN. An Interactive Intelligent System for Crystal Structure Analysis. Enraf-Nonius, Delft, The Netherlands.

Flack, H. D. (1983). Acta Cryst. A39, 876-881.

Kagan, H. (1979). Organic Stereochemistry. London: Edward Arnold.

Kitson, T. M. (1989). Biochem. J. 257, 579-584.

Kitson, T. M. \& Freeman, G. H. (1993). Bioorg. Chem. 21, 354-365.

Kitson, T. M. \& Kitson, K. E. (1994). Biochem. J. 300, 25-30.

Sheldrick, G. M. (1985). SHELXS86. Program for the Solution of Crystal Structures. University of Göttingen, Germany.

\author{
William P. SChaefer, ${ }^{a}$ LaWrence M. Henling, ${ }^{a}$ \\ HENRY C. MCBAY ${ }^{b} \dagger$ AND JOHN ABULU ${ }^{b}$ \\ ${ }^{a}$ Beckman Institute, California Institute of Technology, \\ Pasadena, California 91125, USA, and ${ }^{b}$ Department of \\ Chemistry, Clark-Atlanta University, Atlanta, Georgia 30314. \\ USA
}

(Received 11 January 1995: accepted 27 July 1995)

\section{Abstract}

The dimer of 1-indanolyl obtained from the UV photolysis of 1-indanone in 2-propanol is trans- $\alpha, \alpha^{\prime}$-biindanyl$\alpha, \alpha^{\prime}$-diol, $\left(\mathrm{C}_{9} \mathrm{H}_{9} \mathrm{O}\right)_{2}$. There are two almost identical molecules in the asymmetric unit linked by a hydrogen-bonding system that extends along the $a$ axis. Bond distances and angles are as expected.

\section{Comment}

The diol dimer described here, (I), was prepared in the hope that intramolecular hydrogen bonding would lock it in the correct conformation for enantioselective rearrangement during pinacol rearrangement to produce a spiro compound with a chiral spiro atom. The molecule was the one expected, but all the hydrogen

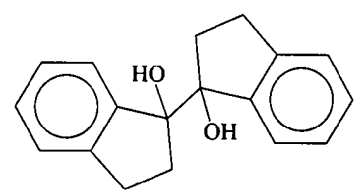

(I)

bonding is intermolecular, this despite IR studies on the compound in solution which indicated intramolecular hydrogen bonding (McBay \& Abulū, 1994). In the crystal, the diol molecules form hydrogen-bonded pairs about centers of symmetry with $\mathrm{O} \cdots \mathrm{O}$ distances $2.716(3)$ and $2.725(3) \AA$; the $\mathrm{O} \cdots \mathrm{H}$ distances are $1.94(3)$ and $1.99(3) \AA$, with $\mathrm{O}-\mathrm{H} \cdots \mathrm{O}$ angles of $152(3)$ and $148(3)^{\circ}$. These pairs are each bonded to

\footnotetext{
† Deceased 23 June 1995.
} 
two non-symmetry-related pairs by two other hydrogen bonds: $\mathrm{O} 1 b-\mathrm{HO} 1 b \cdots \mathrm{O} 2 a$ and $\mathrm{O} 1 a-\mathrm{HO} 1 a \cdots \mathrm{O} 2 b$ $(1+x, y, z)$ with $0 \cdots O$ distances $2.784(3)$ and 2.776 (3) $\AA, \mathrm{H}$. . O distances 2.11 (3) and 2.04 (4) $\AA$ and $\mathrm{O}-\mathrm{H} \cdots \mathrm{O}$ angles $162(4)$ and $163(4)^{\circ}$. This results in a chain of dimers, molecules $a, b, a, b .$. , hydrogen bonded along the $a$ axis with all hydrophilic $\mathrm{O}$ atoms involved (Fig. 2). The exteriors of the chains have the hydrophobic groups showing; there are no interchain hydrogen bonds.

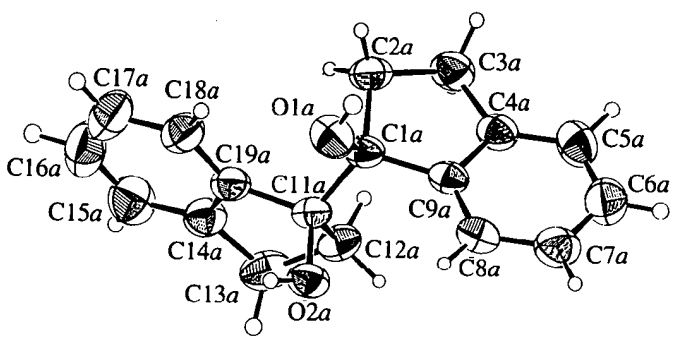

(a)

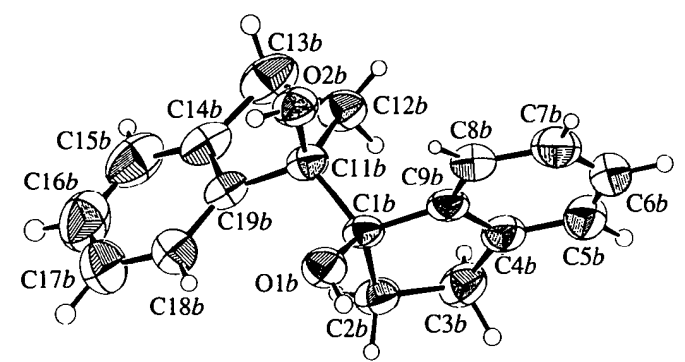

(b)

Fig. 1. ORTEPII (Johnson, 1976) drawings of the two independent molecules showing the atom-numbering system. Non-H atoms are shown as $50 \%$ probability ellipsoids.

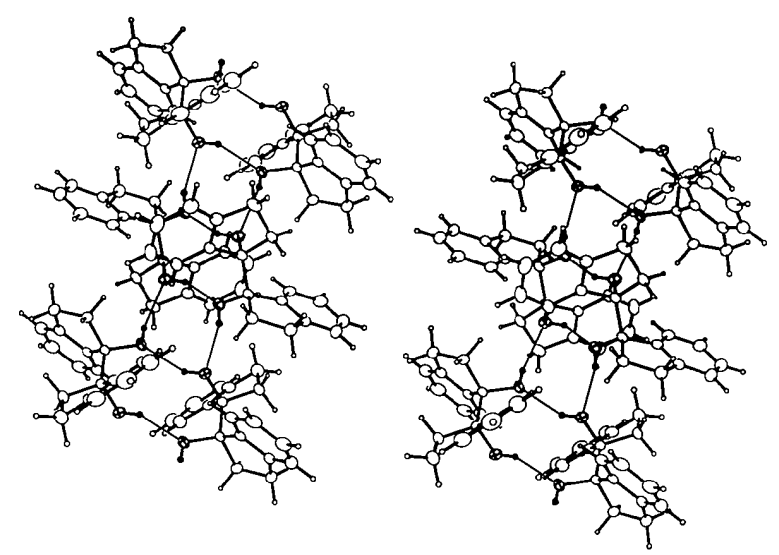

Fig. 2. A stereoview showing the hydrogen bonding, with non-H atoms shown as $20 \%$ probability ellipsoids.

Bond distances and angles are normal when compared with values given by Allen et al. (1987) or, for distances involving the $\mathrm{C}$ atoms linked to $\mathrm{O}$ atoms, with the average of five identical fragments found in the Cambridge Crystallographic Database (Allen, Kennard \& Taylor, 1983). The relevant distances are compared in Table 3, where the values for the present compound are the averages of the four chemically equivalent distances in the two molecules (except there are only two distances for $\mathrm{C} 1-\mathrm{C} 11$ ).

\section{Experimental}

This dimer was the major product obtained by treating 1indanone with magnesium amalgam and $\mathrm{TiCl}_{4}$ in tetrahydrofuran following the procedure outlined by Ahond et al. (1968). (The minor product was trans- $\alpha, \alpha^{\prime}$-dehydrobiindanyl.)

\section{Crystal data}

$\mathrm{C}_{18} \mathrm{H}_{18} \mathrm{O}_{2}$

$M_{r}=266.34$

Triclinic

$P \overline{1}$

$a=10.798$ (3) §

$b=12.100$ (3) $\AA$

$c=12.359(3) \AA$

$\alpha=106.24(2)^{\circ}$

$\beta=104.27(3)^{\circ}$

$\gamma=103.54(2)^{\circ}$

$V=1420.6(6) \AA^{3}$

$Z=4$

$D_{x}=1.25 \mathrm{Mg} \mathrm{m}^{-3}$

\section{Data collection}

$\begin{array}{ll}\text { Enraf-Nonius CAD-4 } & R_{\text {int }}=0.047 \\ \quad \text { diffractometer } & \theta_{\max }=25^{\circ} \\ \omega \text { scans } & h=-12 \rightarrow 12 \\ \text { Absorption correction: } & k=-14 \rightarrow 14 \\ \quad \text { none } & l=-14 \rightarrow 14 \\ 10095 \text { measured reflections } & 3 \text { standard reflections } \\ 4995 \text { independent reflections } & \text { frequency: } 150 \text { min } \\ 4995 \text { observed reflections } & \text { intensity decay: within } \\ \text { [All reflections used, } F_{o}^{2} & \text { counting statistics }\end{array}$

\author{
Mo $K \alpha$ radiation \\ $\lambda=0.71073 \AA$ \\ Cell parameters from 25 \\ reflections \\ $\theta=7-11^{\circ}$ \\ $\mu=0.074 \mathrm{~mm}^{-1}$ \\ $T=294 \mathrm{~K}$ \\ Thin needle \\ $0.52 \times 0.12 \times 0.10 \mathrm{~mm}$ \\ Clear
}

\author{
Refinement \\ Refinement on $F^{2}$ \\ $R(F)=0.043$ for 2646 \\ reflections with \\ $F_{o}^{2}>3 \sigma\left(F_{o}^{2}\right)$ \\ $w R\left(F^{2}\right)=0.008$ on $F^{2}$ for \\ 4995 reflections \\ $S=1.38$ \\ 4995 reflections \\ 505 parameters \\ All $\mathrm{H}$-atom parameters \\ refined
}

$$
\begin{aligned}
& w=1 / \sigma^{2}\left(F_{o}^{2}\right) \\
& (\Delta / \sigma)_{\max }=<0.02 \\
& \Delta \rho_{\max }=0.31 \AA^{-3} \\
& \Delta \rho_{\min }=-0.30 \AA^{-3} \\
& \text { Extinction correction: none } \\
& \text { Atomic scattering factors } \\
& \quad \text { from International Tables } \\
& \text { for X-ray Crystallography } \\
& \quad(1974, \text { Vol. IV) }
\end{aligned}
$$


Table 1. Fractional atomic coordinates and equivalent isotropic displacement parameters $\left(\AA^{2}\right)$

\begin{tabular}{|c|c|c|c|c|}
\hline \multicolumn{5}{|c|}{$U_{\mathrm{eq}}=(1 / 3) \Sigma_{i} \Sigma_{j} U_{i j} a_{i}^{*} a_{j}^{*} \mathbf{a}_{i} \cdot \mathbf{a}_{j}$} \\
\hline & $x$ & $y$ & $z$ & $U_{\text {eq }}$ \\
\hline $\mathrm{Cla}$ & $0.6926(2)$ & $0.1845(2)$ & $0.1995(2)$ & $0.0424(6)$ \\
\hline $\mathrm{C} 2 a$ & 0.8155 (3) & $0.2109(3)$ & $0.3109(3)$ & $0.0539(7)$ \\
\hline $\mathrm{C} 3 a$ & $0.8716(3)$ & $0.3489(3)$ & 0.3782 & $0.0594(9)$ \\
\hline $\mathrm{C} 4 a$ & $0.8086(2)$ & $0.4002(2)$ & $0.2891(2)$ & $0.0470(7)$ \\
\hline $\mathrm{C} 5 a$ & $0.8426(3)$ & $0.5198(3)$ & $0.2941(3)$ & $0.0587(8)$ \\
\hline $\mathrm{C} 6 a$ & $0.7728(3)$ & $0.5468(3)$ & $0.2011(3)$ & $0.0649(9)$ \\
\hline $\mathrm{C} 7 a$ & $0.6706(3)$ & 0.4559 (3) & 0.1038 & $0.0622(8)$ \\
\hline $\mathrm{C} 8 a$ & $0.6375(3)$ & $0.3362(3)$ & $0.0977(2)$ & $0.0508(7)$ \\
\hline $\mathrm{C} 9 a$ & $0.7068(2)$ & $0.3091(2)$ & $0.1915(2)$ & $0.0423(6)$ \\
\hline C11a & $0.5573(2)$ & $0.1221(2)$ & $0.2115(2)$ & $0.0412(6)$ \\
\hline $\mathrm{C} 12 a$ & $0.5234(3)$ & $0.1992(3)$ & $0.3148(2)$ & $0.0504(7)$ \\
\hline $\mathrm{C} 13 a$ & $0.4271(3)$ & $0.1062(3)$ & $0.3440(3)$ & $0.0629(8)$ \\
\hline $\mathrm{C} 14 a$ & $0.4744(2)$ & $-0.0026(2)$ & $0.3133(2)$ & $0.0529(7)$ \\
\hline $\mathrm{C} 15 a$ & $0.4514(3)$ & -0.1018 & 0.3499 (3) & $0.0709(9)$ \\
\hline $\mathrm{Cl} 6 \mathrm{a}$ & $0.5044(4)$ & $-0.1923(3)$ & $0.3093(3)$ & $0.0792(10)$ \\
\hline $\mathrm{C} 17 a$ & 0.5784 (3) & $-0.1845(3)$ & $0.2346(3)$ & 0.0728 \\
\hline $\mathrm{Cl} 18 a$ & 0.6007 (3) & $-0.0866(3)$ & $0.1969(2)$ & $0.0561(7)$ \\
\hline $\mathrm{Cl} 19 a$ & $0.5485(2)$ & $0.0053(2)$ & $0.2373(2)$ & $0.0453(6)$ \\
\hline Ola & $0.6989(2)$ & $0.1024(2)$ & $0.0933(2)$ & $0.0584(5)$ \\
\hline $\mathrm{O} 2 a$ & $0.4494(2)$ & $0.0994(2)$ & $0.1036(2)$ & $0.0501(5)$ \\
\hline $\mathrm{Cl} b$ & $0.1758(2)$ & $0.2368(2)$ & $0.0924(2)$ & $0.0406(6)$ \\
\hline $\mathrm{C} 2 b$ & $0.2819(3)$ & $0.3288(2)$ & $0.0684(3)$ & $0.0508(7)$ \\
\hline $\mathrm{C} 3 b$ & 0.3380 & 0.4478 & $0.1767(3)$ & $0.0605(8)$ \\
\hline $\mathrm{C} 4 b$ & $0.2957(2)$ & $0.4110(2)$ & $0.2721(2)$ & 0.0518 \\
\hline $\mathrm{C} 5 b$ & $0.3394(3)$ & 0.4767 (3) & $0.3940(3)$ & $0.0689(10)$ \\
\hline$C 6 b$ & $0.2955(3)$ & $0.4231(4)$ & $0.4677(3)$ & $0.0764(11)$ \\
\hline $\mathrm{C} 7 b$ & $0.2084(3)$ & $0.3050(3)$ & $0.4223(3)$ & $0.0683(9)$ \\
\hline $\mathrm{C} 8 b$ & $0.1625(3)$ & 0.2387 (3) & $0.3001(3)$ & 0.0528 \\
\hline $\mathrm{C} 9 b$ & $0.2064(2)$ & $0.2930(2)$ & $0.2257(2)$ & $0.0426(6)$ \\
\hline $\mathrm{C} 11 b$ & $0.0292(2)$ & $0.2162(2)$ & $0.0165(2)$ & $0.0431(6)$ \\
\hline $\mathrm{Cl} 2 b$ & $-0.0123(3)$ & 0.3308 & $0.0458(3)$ & $0.0565(8)$ \\
\hline $\mathrm{Cl} 3 b$ & -0.1159 (3) & $0.3180(3)$ & $-0.0709(3)$ & $0.0757(10)$ \\
\hline $\mathrm{Cl} 4 b$ & -0.0770 & $0.2399(3)$ & $-0.1657(3)$ & 0.0621 \\
\hline $\mathrm{C} 15 b$ & -0.1102 & $0.2229(4)$ & $-0.2870(4)$ & 0.0839 (11) \\
\hline $\mathrm{C} 16 b$ & $-0.0647(4)$ & 0.1451 (4) & $-0.3582(4)$ & 0.0954 (13) \\
\hline $\mathrm{C} 17 b$ & $0.0134(4)$ & $0.0834(4)$ & $-0.3115(3)$ & $0.0835(11)$ \\
\hline $\mathrm{C} 18 b$ & 0.0488 (3) & 0.1009 (3) & -0.1909 (3) & $0.0622(8)$ \\
\hline $\mathrm{C} 19 b$ & $0.0040(2)$ & $0.1799(2)$ & $-0.1176(2)$ & 0.0483 (7) \\
\hline Olb & $0.1911(2)$ & $0.1182(2)$ & $0.0608(2)$ & $0.0529(5)$ \\
\hline $\mathrm{O} 2 b$ & $-0.0627(2)$ & $0.1269(2)$ & 0.0409 (2) & $0.0538(5)$ \\
\hline
\end{tabular}

Table 2. Selected geometric parameters $\left(\AA^{\circ},{ }^{\circ}\right)$

\begin{tabular}{|c|c|c|c|}
\hline $\mathrm{C} 1 a-\mathrm{C} 2 a$ & $1.553(4)$ & $\mathrm{Cl} b-\mathrm{C} 2 b$ & $1.548(4)$ \\
\hline $\mathrm{Cl} a-\mathrm{C} 9 a$ & 1.514 (3) & $\mathrm{Cl} b-\mathrm{C} 9 b$ & $1.512(3)$ \\
\hline $\mathrm{Cla}-\mathrm{Clla}$ & $1.542(3)$ & $\mathrm{C} 1 b-\mathrm{C} 11 b$ & $1.549(3)$ \\
\hline $\mathrm{Cla}-\mathrm{O} 1 a$ & $1.436(3)$ & $\mathrm{Cl} b-\mathrm{Ol} b$ & 1.439 \\
\hline $\mathrm{C} 2 a-\mathrm{C} 3 a$ & $1.533(4)$ & $\mathrm{C} 2 b-\mathrm{C} 3 b$ & $1.532(4)$ \\
\hline $\mathrm{C} 3 a-\mathrm{C} 4 a$ & $1.507(4)$ & $\mathrm{C} 3 b-\mathrm{C} 4 b$ & $1.504(4)$ \\
\hline $\mathrm{C} 4 a-\mathrm{C} 5 a$ & 1.387 (4) & $\mathrm{C} 4 b-\mathrm{C} 5 b$ & $1.388(4)$ \\
\hline $\mathrm{C} 4 a-\mathrm{C} 9 a$ & $1.383(3)$ & $\mathrm{C} 4 b-\mathrm{C} 9 b$ & $1.386(4)$ \\
\hline $\mathrm{C} 5 a-\mathrm{C} 6 a$ & $1.376(5)$ & $\mathrm{C} 5 b-\mathrm{C} 6 b$ & $1.369(5)$ \\
\hline $\mathrm{C} 6 a-\mathrm{C} 7 a$ & $1.383(5)$ & $\mathrm{C} 6 b-\mathrm{C} 7 b$ & $1.380(5)$ \\
\hline $\mathrm{C} 7 a-\mathrm{C} 8 a$ & $1.384(4)$ & $\mathrm{C} 7 b-\mathrm{C} 8 b$ & $1.392(5)$ \\
\hline $\mathrm{C} 8 a-\mathrm{C} 9 a$ & $1.383(4)$ & $\mathrm{C} 8 b-\mathrm{C} 9 b$ & $1.381(4)$ \\
\hline $\mathrm{C} 11 a-\mathrm{C} 12 a$ & $1.536(4)$ & $\mathrm{C} 11 b-\mathrm{C} 12 b$ & $1.531(4)$ \\
\hline $\mathrm{C} 11 a-\mathrm{C} 19 a$ & $1.520(3)$ & $\mathrm{C} 11 b-\mathrm{C} 19 b$ & $1.526(4)$ \\
\hline $\mathrm{C} 11 a-02 a$ & $1.445(3)$ & $\mathrm{C} 11 b-\mathrm{O} 2 b$ & $1.438(3)$ \\
\hline $\mathrm{C} 12 a-\mathrm{C} 13 a$ & $1.530(5)$ & $\mathrm{C} 12 b-\mathrm{C} 13 b$ & $1.534(5)$ \\
\hline $\mathrm{C} 13 a-\mathrm{C} 14 a$ & $1.506(4)$ & $\mathrm{C} 13 b-\mathrm{C} 14 b$ & $1.501(5)$ \\
\hline C14a-C15a & $1.388(4)$ & $\mathrm{C} 14 b-\mathrm{C} 15 b$ & $1.395(5)$ \\
\hline $\mathrm{C} 14 a-\mathrm{C} 19 a$ & $1.385(4)$ & $\mathrm{C} 14 b-\mathrm{C} 19 b$ & $1.389(4)$ \\
\hline $\mathrm{C} 15 a-\mathrm{C} 16 a$ & $1.381(5)$ & $\mathrm{C} 15 b-\mathrm{C} 16 b$ & $1.366(6)$ \\
\hline $\mathrm{C} 16 a-\mathrm{C} 17 a$ & $1.370(5)$ & $\mathrm{C} 16 b-\mathrm{C} 17 b$ & $1.377(6)$ \\
\hline $\mathrm{C} 17 a-\mathrm{C} 18 a$ & $1.382(5)$ & $\mathrm{C} 17 b-\mathrm{C} 18 b$ & $1.385(5)$ \\
\hline $\mathrm{C} 18 a-\mathrm{C} 19 a$ & $1.388(4)$ & $\mathrm{C} 18 b-\mathrm{C} 19 b$ & $1.384(4)$ \\
\hline $\mathrm{HO} 2 a \cdots \mathrm{Ol}^{\prime i}$ & $1.94(3)$ & $\mathrm{HO} 2 b \cdots \mathrm{O} b^{\prime i i i}$ & $1.99(3)$ \\
\hline $\mathrm{HO} 1 a \cdots \mathrm{O}^{\prime} b^{\prime \prime \prime}$ & $2.04(4)$ & $\mathrm{HO} 1 b \cdots 02 a^{\prime}$ & $2.11(3)$ \\
\hline $\mathrm{C} 9 a-\mathrm{C} 1 a-\mathrm{C} 2 a$ & $102.8(2)$ & $\mathrm{C} 9 b-\mathrm{Cl} b-\mathrm{C} 2 b$ & $102.8(2)$ \\
\hline $\mathrm{C} 11 a-\mathrm{Cl} a-\mathrm{C} 2 a$ & $112.3(2)$ & $\mathrm{C} 11 b-\mathrm{C} 1 b-\mathrm{C} 2 b$ & $112.4(2)$ \\
\hline $\mathrm{O} 1 a-\mathrm{Cl} a-\mathrm{C} 2 a$ & $111.7(2)$ & $\mathrm{O} 1 b-\mathrm{C} 1 b-\mathrm{C} 2 b$ & $111.7(2)$ \\
\hline
\end{tabular}

\begin{tabular}{|c|c|c|c|}
\hline $\mathrm{C} 11 a-\mathrm{Cl} a-\mathrm{C} 9 a$ & $114.3(2)$ & $\mathrm{C} 11 b-\mathrm{Cl} b-\mathrm{C} 9 b$ & $114.0(2)$ \\
\hline $\mathrm{O} 1 a-\mathrm{Cl} a-\mathrm{C} 9 a$ & $110.7(2)$ & $\mathrm{O} b-\mathrm{Cl} b-\mathrm{C} 9 b$ & $111.0(2)$ \\
\hline $\mathrm{O} 1 a-\mathrm{Cl} a-\mathrm{Cll} a$ & $105.3(2)$ & $\mathrm{O} l b-\mathrm{Cl} b-\mathrm{Cl} b$ & $105.0(2)$ \\
\hline $\mathrm{C} 3 a-\mathrm{C} 2 a-\mathrm{Cl} a$ & $107.7(2)$ & $\mathrm{C} 3 b-\mathrm{C} 2 b-\mathrm{C} 1 b$ & $107.8(2)$ \\
\hline $\mathrm{C} 4 a-\mathrm{C} 3 a-\mathrm{C} 2 a$ & $104.0(2)$ & $\mathrm{C} 4 b-\mathrm{C} 3 b-\mathrm{C} 2 b$ & $103.7(2)$ \\
\hline $\mathrm{C} 5 a-\mathrm{C} 4 a-\mathrm{C} 3 a$ & $128.5(2)$ & $\mathrm{C} 5 b-\mathrm{C} 4 b-\mathrm{C} 3 b$ & $128.5(3)$ \\
\hline $\mathrm{C} 9 a-\mathrm{C} 4 a-\mathrm{C} 3 a$ & $111.0(2)$ & $C 9 b-C 4 b-C 3 b$ & $111.4(2)$ \\
\hline $\mathrm{C} 9 a-\mathrm{C} 4 a-\mathrm{C} 5 a$ & $120.5(2)$ & $\mathrm{C} 9 b-\mathrm{C} 4 b-\mathrm{C} 5 b$ & $120.0(3)$ \\
\hline $\mathrm{C} 6 a-\mathrm{C} 5 a-\mathrm{C} 4 a$ & 119.1 (3) & $\mathrm{C} 6 b-\mathrm{C} 5 b-\mathrm{C} 4 b$ & $119.4(3)$ \\
\hline $\mathrm{C} 7 a-\mathrm{C} 6 a-\mathrm{C} 5 a$ & $120.5(3)$ & $\mathrm{C} 7 b-\mathrm{C} 6 b-\mathrm{C} 5 b$ & $120.8(3)$ \\
\hline $\mathrm{C} 8 a-\mathrm{C} 7 a-\mathrm{C} 6 a$ & $120.7(3)$ & $\mathrm{C} 8 b-\mathrm{C} 7 b-\mathrm{C} 6 b$ & $120.3(3)$ \\
\hline $\mathrm{C} 9 a-\mathrm{C} 8 a-\mathrm{C} 7 a$ & $118.9(3)$ & $\mathrm{C} 9 b-\mathrm{C} 8 b-\mathrm{C} 7 b$ & $118.8(3)$ \\
\hline $\mathrm{C} 4 a-\mathrm{C} 9 a-\mathrm{Cla}$ & 112.1 (2) & $\mathrm{C} 4 b-\mathrm{C} 9 b-\mathrm{Cl} b$ & 111.4 (2) \\
\hline $\mathrm{C} 8 a-\mathrm{C} 9 a-\mathrm{Cla}$ & $127.4(2)$ & $\mathrm{C} 8 b-\mathrm{C} 9 b-\mathrm{Cl} b$ & $127.9(2)$ \\
\hline $\mathrm{C} 8 a-\mathrm{C} 9 a-\mathrm{C} 4 a$ & 120.4 (2) & $\mathrm{C} 8 b-\mathrm{C} 9 b-\mathrm{C} 4 b$ & $120.6(2)$ \\
\hline $\mathrm{C} 12 a-\mathrm{Cl} a-\mathrm{Cl} a$ & $114.4(2)$ & $\mathrm{C} 12 b-\mathrm{C} 11 b-\mathrm{Cl} b$ & $113.3(2)$ \\
\hline $\mathrm{C} 19 a-\mathrm{C} 11 a-\mathrm{Cl} a$ & $115.1(2)$ & $\mathrm{C} 19 b-\mathrm{Cl1} b-\mathrm{Cl} b$ & $114.3(2)$ \\
\hline $\mathrm{O} 2 a-\mathrm{Cll} a-\mathrm{Cla}$ & $108.4(2)$ & $\mathrm{O} 2 b-\mathrm{C} 11 b-\mathrm{C} 1 b$ & $109.0(2)$ \\
\hline $\mathrm{C} 19 a-\mathrm{C} 11 a-\mathrm{C} 12 a$ & $102.3(2)$ & $\mathrm{C} 19 b-\mathrm{C} 11 b-\mathrm{C} 12 b$ & $102.8(2)$ \\
\hline $\mathrm{O} 2 a-\mathrm{C} 11 a-\mathrm{C} 12 a$ & $105.9(2)$ & $\mathrm{O} 2 b-\mathrm{Cl} b-\mathrm{C} 12 b$ & $106.4(2)$ \\
\hline $\mathrm{O} 2 a-\mathrm{Cll} a-\mathrm{C} 19 a$ & $110.2(2)$ & $\mathrm{O} 2 b-\mathrm{C} 11 b-\mathrm{C} 19 b$ & 110.7 (2) \\
\hline $\mathrm{C} 13 a-\mathrm{C} 12 a-\mathrm{C} 11 a$ & $104.5(2)$ & $\mathrm{C} 13 b-\mathrm{Cl} 2 b-\mathrm{C} 11 b$ & $105.5(2)$ \\
\hline $\mathrm{Cl} 4 a-\mathrm{Cl} 3 a-\mathrm{Cl} 2 a$ & $102.5(3)$ & $\mathrm{C} 14 b-\mathrm{C} 13 b-\mathrm{C} 12 b$ & $103.3(3)$ \\
\hline $\mathrm{C} 15 a-\mathrm{Cl} 4 a-\mathrm{C} 13 a$ & $128.7(3)$ & $\mathrm{C} 15 b-\mathrm{C} 14 b-\mathrm{Cl} 3 b$ & $129.4(3)$ \\
\hline $\mathrm{C} 19 a-\mathrm{Cl} 4 a-\mathrm{Cl} 3 a$ & $110.5(2)$ & $\mathrm{Cl} 19 b-\mathrm{Cl} 4 b-\mathrm{Cl} 3 b$ & $110.6(3)$ \\
\hline $\mathrm{C} 19 a-\mathrm{C} 14 a-\mathrm{Cl} 5 a$ & $120.7(3)$ & $\mathrm{C} 19 b-\mathrm{Cl} 14 b-\mathrm{Cl} 5 b$ & $120.0(3)$ \\
\hline $\mathrm{C} 16 a-\mathrm{C} 15 a-\mathrm{C} 14 a$ & $118.7(3)$ & $\mathrm{C} 16 b-\mathrm{C} 15 b-\mathrm{C} 14 b$ & $119.5(4)$ \\
\hline $\mathrm{C} 17 a-\mathrm{C} 16 a-\mathrm{C} 15 a$ & $120.8(3)$ & $\mathrm{C} 17 b-\mathrm{Cl} 16 b-\mathrm{Cl} 15 b$ & $120.8(4)$ \\
\hline $\mathrm{C} 18 a-\mathrm{C} 17 a-\mathrm{Cl} 16 a$ & 120.9 & $\mathrm{C} 18 b-\mathrm{C} 17 b-\mathrm{Cl} 6 b$ & $120.3(4)$ \\
\hline $\mathrm{C} 19 a-\mathrm{Cl} 18 a-\mathrm{C} 17 a$ & $118.9(3)$ & $\mathrm{C} 19 b-\mathrm{C} 18 b-\mathrm{C} 17 b$ & $119.6(3)$ \\
\hline $\mathrm{Cl} 4 a-\mathrm{Cl} 9 a-\mathrm{Cll} a$ & $109.6(2)$ & $\mathrm{C} 14 b-\mathrm{C} 19 b-\mathrm{C} 11 b$ & $110.0(2)$ \\
\hline $\mathrm{C} 18 a-\mathrm{C} 19 a-\mathrm{C} 11 a$ & $130.4(2)$ & $\mathrm{C} 18 b-\mathrm{C} 19 b-\mathrm{C} 11 b$ & $130.1(2)$ \\
\hline $\mathrm{C} 18 a-\mathrm{Cl} 19 a-\mathrm{Cl} 4 a$ & $119.9(2)$ & $\mathrm{Cl} 18 b-\mathrm{Cl} 19 b-\mathrm{Cl} 4 b$ & $119.8(3)$ \\
\hline $\mathrm{O} 2 a-\mathrm{HO} 2 a \cdots \mathrm{O} 1 a^{\prime i}$ & $152(3)$ & $\mathrm{O} 1 b-\mathrm{HO} 1 b \cdots \mathrm{O} 2 a^{\prime}$ & $162(4)$ \\
\hline $\mathrm{O} 1 a-\mathrm{HO} 1 a \cdots \mathrm{O} 2 b^{\prime \prime \prime}$ & $163(4)$ & $\mathrm{O} 2 b-\mathrm{HO} 2 b \cdots \mathrm{O} 1 b^{\prime \prime \prime \prime}$ & $148(3)$ \\
\hline
\end{tabular}

Symmetry codes: (i) $1-x,-y,-z$; (ii) $1+x, y, z$; (iii) $-x,-y,-z$.

Table 3. Comparison of observed and expected bond distances $(\AA)$

$\begin{array}{llll}\mathrm{C} 1,11 & \mathrm{C} 2,12 & 1.542(10)^{*} & 1.530 \\ \mathrm{C} 1,11 & \mathrm{C} 9.19 & 1.518(6) & 1.547 \\ \mathrm{C} 1,11 & \mathrm{O} 1,2 & 1.440(4) & 1.432 \\ \mathrm{Cl} & \mathrm{C} 11 & 1.546(4) & 1.546 \\ \mathrm{C} 2,12 & \mathrm{C} 3,13 & 1.532(2) & 1.534 \\ \mathrm{C} 3,13 & \mathrm{C} 4,14 & 1.505(3) & 1.510 \\ \mathrm{C} 4,8,14,18 & \mathrm{C} 5,9,15,19 & 1.387(4) & 1.387 \\ \mathrm{C} 4,14 & \mathrm{C} 9,19 & 1.386(3) & 1.397 \\ \mathrm{C} 5,6,7,15,16,17 & \mathrm{C} 6,7,8,16,17,18 & 1.379(7) & 1.380\end{array}$

* Numbers in parentheses are the sample standard deviations.

The structure was solved by MULTAN (Debaerdemaeker $e t$ al., 1988); all heavy atoms were found in the $E$ map. Their positions and isotropic displacement parameters were refined by least squares and a subsequent difference map showed peaks for most of the $\mathrm{H}$ atoms. The $\mathrm{H}$ atoms on the $\mathrm{C}$ atoms were initially placed by calculation; however, the final refinement included positional parameters for all atoms, anisotropic displacement parameters for the heavy atoms, isotropic displacement parameters for the $\mathrm{H}$ atoms and a scale factor, leading to the values reported here.

Data collection: CAD-4 Software (Enraf-Nonius, 1989). Cell refinement: CAD-4 Software. Data reduction: CRYM (Duchamp, 1964). Program(s) used to solve structure: $C R Y M$. Program(s) used to refine structure: CRYM. Molecular graphics: ORTEPII (Johnson, 1976). Software used to prepare material for publication: $C R Y M$. 
Lists of structure factors, anisotropic displacement parameters, $\mathrm{H}$ atom coordinates and complete geometry have been deposited with the IUCr (Reference: SZ1046). Copies may be obtained through The Managing Editor, International Union of Crystallography, 5 Abbey Square, Chester CH1 2HU, England.

\section{References}

Ahond, A., Caré, A., Kan-Fan, C., Husson, H.-P., de Rostolan, J. \& Potier, P. (1968). J. Am. Chem. Soc. 90, 5622-5623.

Allen, F. H., Kennard, O. \& Taylor, R. (1983). Acc. Chem. Res. 16, 146-153.

Allen, F. H., Kennard, O., Watson, D. G., Brammer, L., Orpen, A. G. \& Taylor, R. (1987). J. Chem. Soc. Perkin Trans. pp. S1-19.

Debaerdemaeker, T., Germain, G., Main, P., Refaat, L.S., Tate, C. \& Woolfson, M. M. (1988). MULTAN88. A System of Computer Programs for the Automatic Solution of Crystal Structures from $X$ ray Diffraction Data. Universities of York, England, and Louvain, Belgium.

Duchamp, D. J. (1964). Am. Crystallogr. Assoc. Meet., Bozeman, Montana, Paper B14, p. 29.

Enraf-Nonius (1989). CAD-4 Software. Version 5.0. Enraf-Nonius, Delft, The Netherlands.

Johnson, C. K. (1976). ORTEPII. Report ORNL-5138. Oak Ridge National Laboratory, Tennessee, USA.

McBay, H. C. \& Abulū. J. (1994). Unpublished results.

\section{Comment}

The intramolecular Michael reaction has been used successfully for the synthesis of medium sized (nine- and ten-membered) rings (Deslongchamps \& Roy, 1986). Recently, the formation of 14 -membered rings was reported using pseudo-high-dilution techniques (Girard \& Deslongchamps, 1992). In order to verify the relative ease of macrocyclization of five-membered $\beta$-ketoesters with enones and ynones, a series of new 14 -membered ring compounds were synthesized from precursors differing in the nature of their Michael acceptors and in the hybridization state of one $\mathrm{C}$ atom (Berthiaume \& Deslongchamps, 1995). X-ray diffraction analysis of the products was undertaken in order to confirm the structure and relative stereochemistry, not possible by conventional spectroscopic analysis. We report here the crystal structures of the two title 14-membered ring macrocycles, (I) and (II).<smiles>C=C1CCC(=CCC[C@@H]2CCC(=O)[C@]2(CCC(=O)CC)OC)CC1</smiles>

(I)<smiles>C=C1CCC(=CCC[C@H]2CCC(=O)[C@]2(/C=C/C(=O)CC)OC)CC1</smiles>

(II)

\section{4-Membered Macrocycles: Methyl trans- (10E)-5-Methyl-8-methylene-11,15-dioxobi-} cyclo[12.3.0]heptadec-4-ene-14-carboxylate, (I), and Methyl trans-(2E,10E)-10-Methyl7-methylene-4,17-dioxobicyclo[12.3.0]heptadeca-2,10-diene-1-carboxylate, (II)

\author{
Marc Drouin, ${ }^{a}$ Gilles Berthiaume ${ }^{b}$ ANd Pierre \\ DESLONGCHAMPS $^{b}$
}

${ }^{a}$ Laboratoire de Diffraction des Rayons-X, Département de Chimie, Université de Sherbrooke, Sherbrooke, Quebec, Canada JIK $2 R I$, and ${ }^{b}$ Laboratoire de Synthèse Organique, Département de Chimie, Université de Sherbrooke, Sherbrooke, Quebec, Canada JIK 2RI

(Received 3 November 1994; accepted 24 July 1995)

\begin{abstract}
A new series of functionalized 14-membered ring compounds were synthesized by an intramolecular Michael reaction. The structures of the title compounds (I) and (II) $\left(\mathrm{C}_{2 !} \mathrm{H}_{30} \mathrm{O}_{4}\right.$ and $\mathrm{C}_{21} \mathrm{H}_{28} \mathrm{O}_{4}$, respectively) were determined by single-crystal $\mathrm{X}$-ray diffraction in order to confirm their relative stereochemistries.
\end{abstract}

The results show the ring junction to be trans in both molecules and the olefin geometries to be trans in (I) and trans,trans in (II). The global conformations of the molecules are very similar. In both compounds, the methylene moiety is facing the five-membered ring. The methyl carboxylate group is equatorial to the plane defined by the atoms forming the 14 -membered ring, whereas the five-membered ring is axial to this plane. In (I), C20 and C21 are facing down on the same side of the macrocycle as the five-membered ring, whereas the $\mathrm{C} 4=\mathrm{O} 3$ carbonyl group is facing up. In (II), C20 and $\mathrm{C} 21$ are also facing down, whereas the $\mathrm{C} 4=\mathrm{O} 3$ carbonyl group is in the plane of the 14 -membered ring. Puckering analysis on the five-membered ring shows an envelope conformation in (I) and (II). C15 is 0.508 (2) $\AA$ from the plane defined by C1, C14, C16 and $\mathrm{C} 17$ in (I). $\mathrm{C} 14$ is 0.524 (10) $\AA$ from the plane defined by $\mathrm{C} 1, \mathrm{C} 15, \mathrm{C} 16$ and $\mathrm{C} 17$ in (II). In compound (I), there is no transannular close contact. In (II), the $\mathrm{O} 3$ ketone group and $\mathrm{C} 2=\mathrm{C} 3$ trans double bond are conjugated, as shown by the $\mathrm{C} 3-\mathrm{C} 4$ bond distance [1.462 (7) $\AA$ ] and the $\mathrm{O} 3-\mathrm{C} 4-\mathrm{C} 3-\mathrm{C} 2$ torsion angle $\left[2.6(4)^{\circ}\right]$. This particular rigid geometry is such that $\mathrm{H} 3$ 